\title{
A study to assess knowledge andpractice of basic life support among nurses working in tertiary care hospital, New Delhi, India
}

\begin{abstract}
Cardiovascular disease is the world's largest killer. Nurses are often the first to activate life support measures when a cardio-respiratory arrest happens. Hence, they should be wellequipped with knowledge and skills of Cardiopulmonary resuscitation to save the life of patient. The aim of the present study was to assess the knowledge and practice of basic life support among nursing officers working in tertiary care hospital, Delhi, India. A cross sectional survey analysis was carried out among 112 nursing officers working in various departments, in March 2019, using convenient sampling technique. A semi structured questionnaire containing knowledge and skill items were used to assess knowledge and skill related to CPR. The results showed average knowledge and poor skill among subjects. Whereas the subjects working in ICU and emergency departments had better knowledge and performance than other selected areas. Both Knowledge and skill were significantly associated with variables like clinical area, in-service training on BLS at $\mathrm{p}<0.05$. The results strongly recommend the need for regular basic life support training sessions for health care professionals.
\end{abstract}

Keywords: basic life support, cardio respiratory arrest, health care, knowledge, age, sex, qualification, clinical area, clinical experience
Volume 7 Issue 2 - 2020

\author{
Seema Sachdeva \\ Department of Nursing education, College of Nursing, AllMS, \\ India
}

Correspondence: Seema Sachdeva, Department of Nursing education, College of Nursing, AllMS, New Delhi, India, Emailsachdevaaiims@gmail.com

Received: April 15, 2020 | Published: June 30, 2020
Abbreviations: BLS, basic life support; CPR, cardio respiratory arrest ; ILCOR, international liaison committee on resuscitation

\section{Introduction}

Cardiac arrest is a substantial health problem estimated to accoun for approximately 15-20 percent of all deaths in both developing and developed countries. ${ }^{1}$ Cardiopulmonary resuscitation (CPR) is considered a core emergency skill in which all health care professionals must be proficient. Early initiation of cardiopulmonary resuscitation (CPR) and activation of the chain of survival are key factors in the saving life of patients with cardiac arrest. ${ }^{2}$ As the nurses remain with patient round the clock and spend significant time alongside patients they are often the first to realize in hospital cardiac arrest. ${ }^{3}$ Literature indicates differences in Nurses CPR knowledge and skills from their area of work and experience. ${ }^{4}$ In western world and other developing countries too, CPR knowledge and skills differs among health-care workers. ${ }^{4,5}$ In India, there is no established compulsory CPR training schedules and stringent need to renew the license/ certification for basic life support for hospital-based health care providers which results in wide gap in knowledge and practice of CPR. ${ }^{5} \mathrm{Hence}$, in light of international guidelines and recommendations, the researchers have felt the need of assessing knowledge and skills of Basic Life support among nursing officers working in tertiary care centre, Delhi, India. The secondary objective was to find its association with selected variables.

\section{Material and methods}

The Non- experimental descriptive survey was conducted among 112 nursing officers (estimated sample size, $\mathrm{N}=100$ ) selected by using convenient sampling technique in February and March 2019. The study was conducted among full time working nursing officers in various departments like Emergency ward, Medicine ward,
Surgical ward, Intensive care Unit and Operating room. The senior administrative and managerial staff who were not involved in direct patient care and those who did not wish to participate in study were excluded. Tools used were Demographic profile (items like age, sex, qualification, clinical area, clinical experience, in-service BLS training); a 20 items multiple Choice semi-structured questionnaire to assess the knowledge and 12 items skill checklist to assess the practice of BLS (using mannequin in simulation lab) based upon 2015 Basic Life Support guidelines laid by American Heart Association. The main items were related to primary assessment, airway management, positioning, effective chest compression and ventilation and use of automated external defibrillator. Each correct answer was given 1 mark in knowledge questionnaire with total marks 20. A score greater than 15 was categorized under good knowledge, 11-15score under average knowledge and $\leq 10$ under poor knowledge. For skill assessment, coding was done for performance or not performance of the particular step with total marks as 12 . The performance of the step which was not correctly done or missing step was coded as zero while correctly performing the step was marked 1 score. The skill was assessed under two categories i.e. poor skill (scores $\leq 9 ;<75 \%$ ) and good skill (score $>9 ;>75 \%$ ). The tool was validated by 5 experts and reliability was checked using test rest method for knowledge questionnaire $(\mathrm{r}=0.75)$ inter rater reliability for skill checklist $(r=0.82)$. Written informed consent was taken from subjects to conduct the study after obtaining ethical approval from institute. Pilot study was conducted among 15 subjects in cardiothoracic department and study was found feasible. Data collection was done in morning and evening shift as per the convenience and availability of subjects. Self-reported questionnaire was distributed for knowledge and subjects demonstrated BLS procedure on mannequin for assessment of skill. The data was entered in Excel sheet and analyzed using SPSS 15. Descriptive statistics (Frequencies, proportions, mean and standard deviation) were used in data analysis. ANOVA was used to find the association of knowledge 
and skill with selected variables. Level of significance $(\alpha)$ was kept at0.05.

\section{Results}

A total of 112 subjects participated in the study, out of which majority (65\%) had done B.Sc Nursing and were females (51\%). Majority of the subjects were from medicine department (28\%) and surgery department (25\%) followed by emergency department (23\%) and ICU (14\%) and OT (10\%). 56\% subjects had clinical experience of $>5$ years. With regards to in-service education $61 \%$ subjects had never got any training related to basic life support.

For knowledge of subjects regarding BLS, the average score was $12.2 \pm 3.2$ with a range $=5-19$. Majority of the subjects $(68 \%)$ subjects had average level of knowledge as shown in Table 1.

Table I Level of knowledge of subjects for BLS

\begin{tabular}{lll}
\hline Level of knowledge & F (\%) & Mean \pm SD \\
\hline Good $(>15)$ & $22(20)$ & $16.3 \pm 2.1$ \\
Average $(1 \mathrm{I}-15)$ & $76(68)$ & $12.2 \pm 3.2$ \\
Poor $(\leq 10)$ & $14(12)$ & $8.4 \pm 2.04$ \\
\hline
\end{tabular}

Total Scores $=20$

Item wise assessment for knowledge of CPR showed that most of subjects correctly responded for primary assessment (76\%), chain of survival steps $(74 \%)$, hand position in CPR $(90 \%)$, ventilation compression ratio (80\%) whereas there was lack of knowledge for items like cardinal signs of arrest (42\%), carotid pulse assessment $(19 \%)$, change of compressor( $38 \%$ ), location of chest compression( $54 \%)$ and indication of defibrillation(41\%) as shown in Table 2.

Table 2 Frequency distribution of the subjects as per the correct responses in knowledge questionnaire

\begin{tabular}{llll}
\hline S.no & Knowledge items & \multicolumn{2}{c}{ Correct responses } \\
& & F & $\%$ \\
\hline 1 & Indications of basic life support & 75 & 67 \\
3 & Primary assessment & 87 & 76 \\
4 & Cardinal signs of arrest & 47 & 42 \\
5 & Chain of survival steps & 83 & 74 \\
6 & Time of assessing carotid pulse & 21 & 19 \\
7 & Location of chest compression & 61 & 54 \\
8 & Hand position for effective CPR & 101 & 90 \\
9 & Compression Rate & 76 & 68 \\
10 & Compression-ventilation ratio & 90 & 80 \\
11 & Depth of compression & 75 & 67 \\
12 & Chest recoiling & 84 & 74 \\
13 & Nirway maintenance & 70 & 65 \\
\hline
\end{tabular}

\begin{tabular}{lllc}
\multicolumn{2}{l}{ Table Continued } \\
\hline S.no & Knowledge items & \multicolumn{2}{l}{ Correct responses } \\
\hline 14 & Pulse reassessment & 74 & 66 \\
15 & Switch time of compressor & 42 & 38 \\
16 & Indication of Defibrillation & 46 & 41 \\
17 & Pad placement in AED & 62 & 55 \\
18 & Caution to use defibrillator & 90 & 80 \\
19 & Importance of Continued CPR & 95 & 85 \\
20 & Recovery position & 39 & 35
\end{tabular}

$N=112$

With regard to skill for BLS, the average skill scores of the subjects were $7.12 \pm 2.16$ (total scores $=12$ ) with Range $=4-11$. Majority $(64 \%)$ of the subjects showed poor performance I.e. $<9$ as shown in Table 3.

Table 3 Level of skill of study subjects for BLS

\begin{tabular}{lll}
\hline Level of skill & F (\%) & Mean \pm SD \\
\hline Poor $(<9)$ & 7I $(64)$ & $6.33 \pm 1.21$ \\
Good (>9) & 4 I (36) & $9.25 \pm 1.08$
\end{tabular}

Total scores $=12$

In item wise skill assessment, the common incorrect or missed steps were like calling for help (62\%), Quality chest compression (rate, depth and recoiling of chest) $66 \%$, pulse reassessment $(77 \%)$ and correct AED pads placement (62\%) as shown in Figure 1.

With regard to knowledge and skill of subjects posted in different departments, the nursing officers from ICU, emergency department had better knowledge and skill as show in Figure 2.

In terms of association of knowledge and skill of the subjects with demographic variables, there was significant association of knowledge and skill with experience, clinical area and in service education with $p$ value $<0.05$ as shown in Table 4 .

\section{Discussion}

The finding of the study showed that most of the subjects had average knowledge (64\%) and poor skill $(66 \%)$ for basic life support. Most of the subjects had less knowledge about the critical components of CPR likecardinal signs of arrest, carotid pulse assessment, change of compressor, location of chest compression and indication of defibrillation.Skill was found poor in components like Calling for help, quality CPR (rate/ depth and recoiling of chest), pulse reassessment, location of AED pads on chest. The findings of this study concurs with a study done in Bahrain which reported that only $15 \%$ (total subjects 200) of the nurses passed the CPR skills test based on the standard pass mark of $85 .{ }^{6}$ Other similar supporting studies also quoted the lack of knowledge and skill among health care professionals, including nurses and physicians. Basic aspects of CPR, such as the correct compression and ventilation sequence, did not appear to be correctly understood by all healthcare professionals and it was suggested that 
CPR training needs to be improved in medical and nursing schools..$^{5,7}$ Another study that concur with the findings showed that there is a significant gap in theoretical and practical knowledge on CPR among nurses. This gap shows considerable room for improvement. ${ }^{8}$ The present study findings highlighted that emergency area and ICU area nurses had comparatively better knowledge and skill scores than other areas like OT, medicine and surgery department as shown in Figure 2. Other studies with supportive findings reported that nurses working in high-risk areas such as Intensive Care Unit (ICU) and nurses who work continuously in close contact with patients are more motivated to maintain their competence in CPR than other area nurses where there is lack of frequent exposure. ${ }^{9}$ While on the contrary, a recent study conducted in Botswana indicated that regardless of the setting of the hospitals like acute and non acute patient care units, CPR knowledge and skills was found poor among nurses working in different areas after a gap of more than 6 months after initial training in CPR which further reduced nurse's initiation in performance of CPR. ${ }^{10}$ In present study, participants with more experience and who underwent BLS training performed better than nurses who never had any exposure to BLS training programme. This is also supported by recent studies conducted in Indiawhere nurses with higher experience with BLS training performed better than the nurses who never had any training. ${ }^{6,11}$ Another supported findings suggested by Mohsenabadi reports that debriefing-based training promotes improved knowledge and competence in CPR. ${ }^{12}$ In 2010 the International Liaison Committee on Resuscitation (ILCOR) reported thatbasic and advanced lifesupport knowledge and skills are likely to deteriorate over ashort period of time, approximately 3-6 months and it recommends periodic evaluations to identify thoseprofessionals who need to refresh their knowledge and skills. ${ }^{13}$

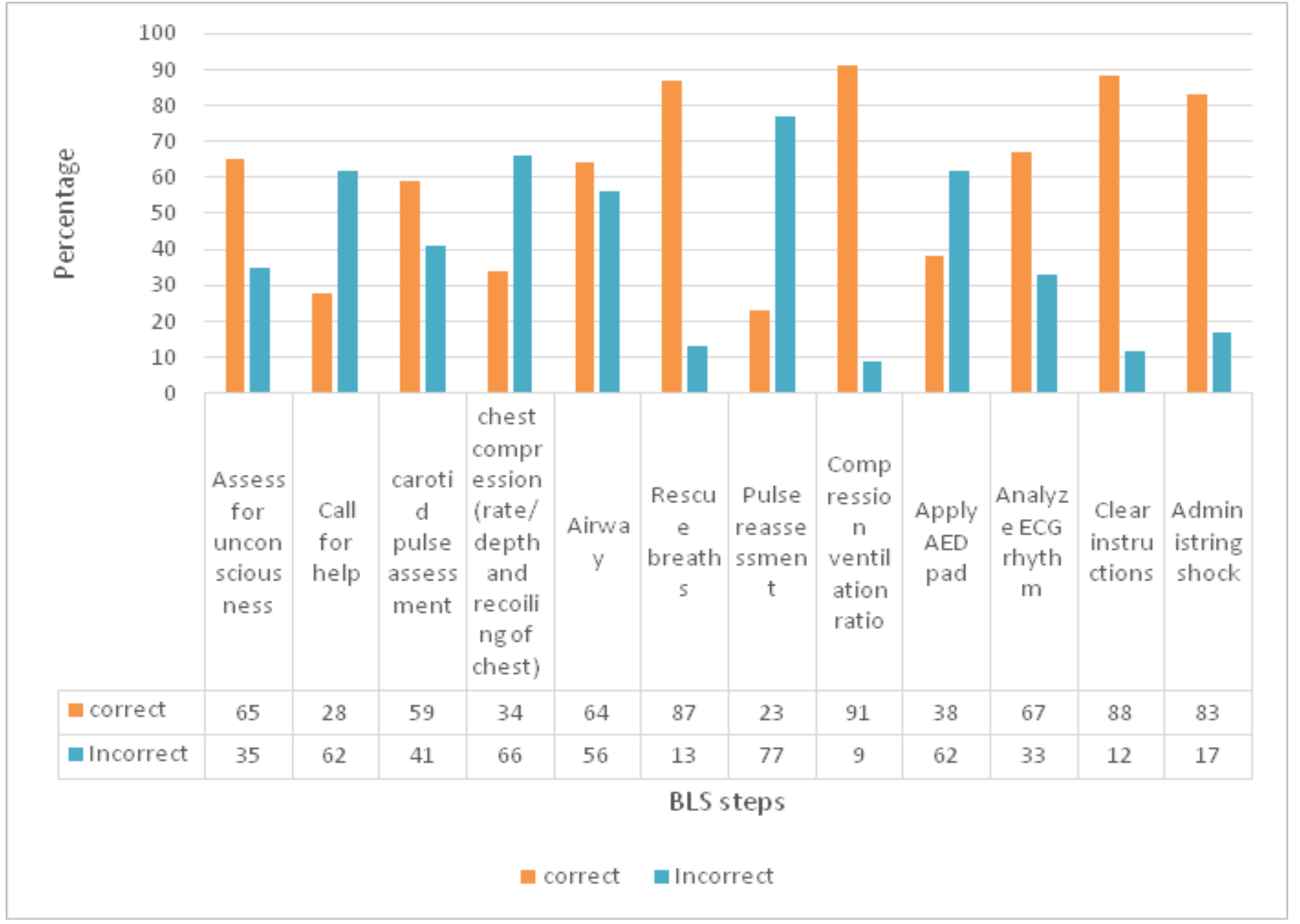

Figure I Percentage wise distribution of correct and in-correct steps in CPR. 


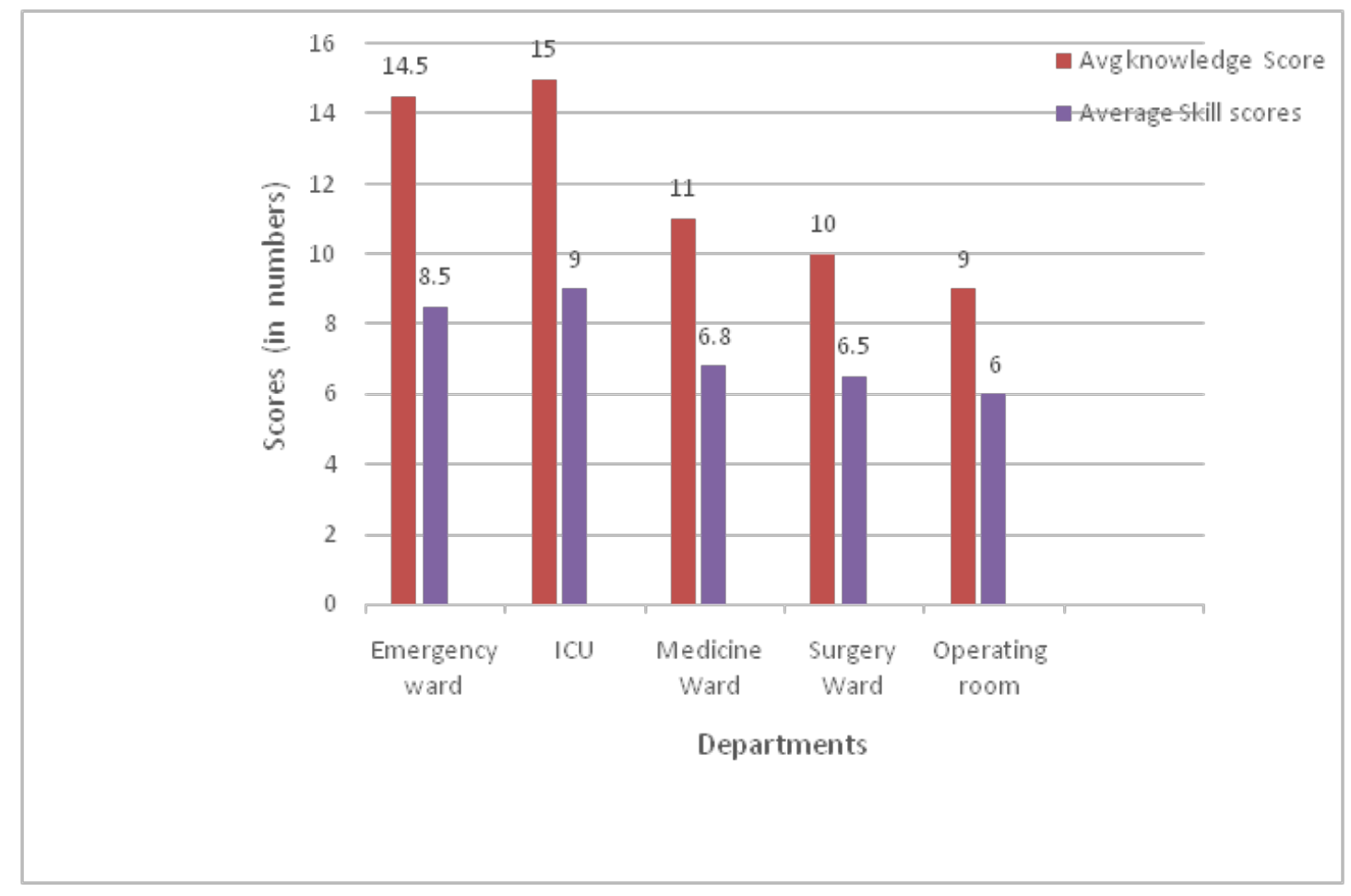

Figure 2 Distribution of Knowledge and skill scores among subjects in different departments.

Table 4 Association of knowledge and skills with the selected variables

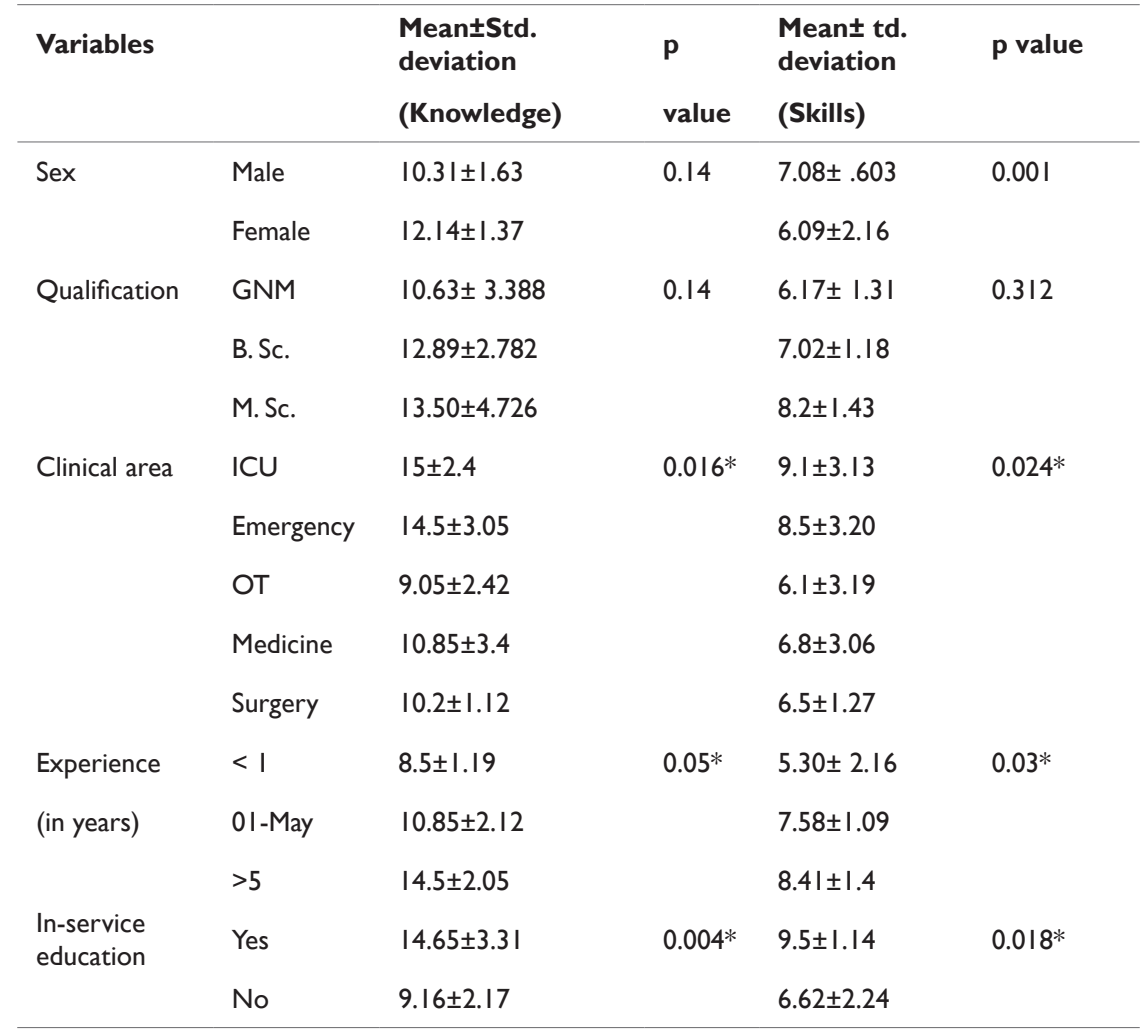

ANOVA test $(p<0.05$ statistically significant) 


\section{Conclusion}

The study showed that majority of the subjects had average knowledge and poor skill for Basic Life Support. Majority of subjects were lacking in critical steps like quality CPR (chest compression rate, depth), carotid pulse assessment, and calling for help and location of AED placement. The staff working in critical areas like emergency ward and Intensive care unit showed better performance than other units like medicine and surgery ward. The main limitations of study were that the non-probability sampling method was used and there was less sample size for generalization of the findings. The practice of BLS was assessed in a simulated environment rather than observing actual performance of subjects in real life situation which can add to the bias.

\section{Funding}

None.

\section{Acknowledgments}

None.

\section{Conflicts of interest}

The author declares that there is no conflict of interest to declare.

\section{References}

1. John Bosco Tamu Munezero et al. Assessment of nurses knowledge and skills following cardiopulmonary resuscitation training at Mbarara Regional Referral Hospital, Uganda. Pan Afr Med Js. 2018;30:108.

2. Bullock Ian. Skill acquisition in resuscitation. Resuscitation. 2000;45(2):139-143.

3. Kabina Ratha, Swarnakanti Panda, Rashmimala Pradhan. Evaluate the Effectiveness of Planned Teaching Programme regarding Basic Life Support (BLS) among Intern (B. Sc Nursing) Student at Selected Nursing College, Bhubaneswar,Odisha. Nurs Health Sci. 2014;3(1):16-19.
4. Passali C, Pantazopoulos I, Dontas I, et al. Evaluation of nurses' and doctors' knowledge of basic and advanced life support resuscitation guidelines. J Nurs Educ Pract. 2011;11(6):365-369.

5. Kidd Tracy, Kendall Sharon. Review of effective advanced cardiac life support training using experiential learning. J Clin Nurs. 2007;16(1):58-66.

6. Marzooq Hussain, Lyneham Joy. Cardiopulmonary resuscitation knowledge among nurses working in Bahrain. Int $J$ Nurs Pract. 2009;15(4):294-302.

7. Sodhi Kanwalpreet, Singla Manender Kumar, Shrivastava Anupam. Impact of advanced cardiac life support training program on the outcome of cardiopulmonary resuscitation in a tertiary care hospital. Indian journal of critical care medicine. Indian J Crit Care Med. 2011;15(4):209-212.

8. Nyman J, Sihvonen M. Cardiopulmonary resuscitation skills in nurses and nursing students. Resuscitation. 2000;47(2):179-184.

9. Verplancke T. Determinants of the quality of basic life support by hospital nurses. Resuscitation. 2008;77(1):75-80.

10. Rajeswaran Lakshmi, Ehlers Valerie J. Cardiopulmonaryresuscitation knowledge and skills of registered nurses in Botswana. Curationis. 2014;37(1):1-7.

11. Saramma PP, Raj LS, Dash PK, et al. Assessment of long-term impact of formal certified cardiopulmonary resuscitation training program among nurses. Indian J Crit Care Med. 2016;20(4):226-32.

12. Mohsenabadi M. The effect of debriefing-based training about cardiopulmonary resuscitation on nurses' knowledge. Resuscitation. 2018;130:e81.

13. Dal U, Sarpkaya D. Knowledge and psychomotor skills of nursing students in North Cyprus in the area of cardiopulmonary resuscitation. Pak J Med Sci. 2013;29(4):966-971. 